Pacific Journal of Mathematics

A RADON-NIKODÝM THEOREM FOR VECTOR AND 


\title{
A RADON-NIKODYM THEOREM FOR VECTOR AND OPERATOR VALUED MEASURES
}

\author{
Jorge Alvarez DE ARAYA
}

The main result of this paper is a Radon-Nikodým theorem for measures taking values in a separable Hilbert space and on the bounded operators of such a space. The integral used for the representation is a Gelfand-Pettis integral, which in this case is also equivalent to the Bochner integral.

1.1. Basic definitions. We will consider the following objects: a measure space $(\Omega, \mathscr{A}, \mu)$, where $\mathscr{A}$ is a $\sigma$-algebra of subsets of $\Omega$ and $\mu$ is a $\sigma$-finite nonnegative measure; a separable Hilbert space $H$ and the space $B(H)$ of bounded linear operators from $H$ into $H$, and also the objects which we define below.

1.2. Definition. By vector function and operator function we will understand functions defined on $\Omega$ and taking values in $H$ and $B(H)$ respectively. A vector function $x(\omega)$ is measurable if for each $y$ in $H$, the function $(y, x(\omega))$ is measurable. An operator function $A(\omega)$ is measurable if for each $x, y$ in $H$, the function $(A(\omega) x, y)$ is measurable. Obviously $A(\omega)$ is measurable if and only if $A(\omega) x$ is a measurable vector function for each $x$ in $H$.

1.3. LEMмA. If $x(\omega)$ is a measurable vector function, then $\|x(\omega)\|$ is measurable. If $A(\omega)$ is a measurable operator function, then $\|A(\omega)\|$ is measurable.

Proof. Let $x(\omega)$ be measurable and let $\left\{e_{1} e_{2}, \cdots\right\}$ denote an orthonormal basis for $H$. Then $\left(x(\omega), e_{n}\right)$ is measurable for each $n$ and so $\|x(\omega)\|^{2} \sum_{n=1}^{\infty}\left|\left(x(\omega), e_{n}\right)\right|^{2}$ is measurable. Now let $A(\omega)$ be measurable and let $S_{0}$ be a countable dense subset of the unit ball in $H$. Then $\|A(\omega)\|=\sup \left\{\|A(\omega) x\|: x \in S_{0}\right\}$ is measurable.

1.4. Definition. A measurable vector function $x(\omega)$ is integrable if $\|x(\omega)\|$ is integrable (i.e., it belongs to $\left.L_{1}(\mu)\right)$. A measurable operator function $A(\omega)$ is integrable if $\|A(\omega)\|$ is integrable.

Let $x(\omega)$ be integrable and let $y \in H$. Then $|(y, x(\omega))| \leqq\|y\| \cdot\|x(\omega)\|$ and $(y, x(\omega))$ is integrable. $\int(y, x(\omega)) d \mu(\omega)$ is a linear functional bounded by $\int\|x(\omega)\| d \mu(\omega)$ and there is a unique vector $z \in H$ such that $\int(y, x(\omega)) d \mu(\omega)=(y, z)$. The vector $z$ is by definition the integral 
$\int x(\omega) d \mu(\omega)$; we already proved that $\left\|\int x(\omega) d \mu(\omega)\right\| \leqq \int\|x(\omega)\| d \mu(\omega)$. The integral is obviously linear. For each

$$
x \in H,\|A(\omega) x\| \leqq\|A(\omega)\| \cdot\|x\|
$$

so that $A(\omega) x$ is an integrable vector function. Since

$$
\left\|\int A(\omega) x d \mu(\omega)\right\| \leqq \int\|A(\omega) x\| d \mu(\omega) \leqq \int\|A(\omega)\| d \mu(\omega) \cdot\|x\|,
$$

$\int A(\omega) x d \mu(\omega)$ defines a bounded linear operator on $x$. This operator is by definition the integral of $A(\omega)$, so that $\int A(\omega) x d \mu(\omega)=\left(\int A(\omega) d \mu(\omega)\right) x$ for each $x \in H$. Obviously $\left\|\int A(\omega) d \mu(\omega)\right\| \leqq \int\|A(\omega)\| d \mu(\omega)$ and the integral is linear.

2.1. Indefinite integrals and the Radon-Nikodým theorem. If $x(\omega)$ is a measurable vector function and $E \in \mathscr{A}, \chi_{E}(\omega) x(\omega)$ is also measurable and if $x(\omega)$ is integrable, so is $\chi_{E}(\omega) x(\omega)$. Similarly, if $A(\omega)$ is an operator function, $\chi_{E}(\omega) A(\omega)$ will be measurable or integrable if $A(\omega)$ has the same property. Thus, if $x(\omega)$ and $A(\omega)$ are integrable, $\int_{E} x(\omega) d \mu(\omega) \equiv \int \chi_{E}(\omega) x(\omega) d \mu(\omega)$ and

$$
\int_{E} A(\omega) d \mu(\omega) \equiv \int \chi_{E}(\omega) A(\omega) d \mu(\omega)
$$

will exist for all $E \in \mathscr{A}$.

Let $\varphi(E)$ denote the integral over $E$ of a vector or operator function. Then $\varphi$ is $\sigma$-additive in norm, that is, if $\left\{E_{n}\right\}_{n=1}^{\infty}$ is a sequence of disjoint sets in $\mathscr{A}$, then $\varphi\left(\bigcup_{n=1}^{\infty} E_{n}\right)=\sum_{n=1}^{\infty} \varphi\left(E_{n}\right)$ in norm. Also $\phi$ is absolutely continuous with respect to $\mu(\varphi \ll \mu)$ in the sense that $(\mu E)=0$ implies $\varphi(E)=0$. Finally if $E \in \mathscr{A}$ and $\left\{E_{n}\right\}_{n=1}^{\infty}$ is a disjoint sequence of sets in $\mathscr{A}$ such that $E=\bigcup_{n=1}^{\infty} E_{n}$, then we must have $\sum_{n=1}^{\infty}\left\|\varphi\left(E_{n}\right)\right\|<\infty$. We will denote this property saying that is $\sigma$ bounded on $E$.

2.2. Lemma. Let $X$ be a normed space and $\varphi$ a $\sigma$-additive function from $\mathscr{A}$ into $X$. Then there is a nonnegative measure $\nu$ on $\mathscr{A}$ such that for each $E \in \mathscr{A},\|\varphi(E)\| \leqq \nu(E)$, and $\nu(E)$ is finite if and only if $\varphi$ is $\sigma$-bounded on $E$. Furthermore if $\varphi \ll \mu$, then $\nu \ll \mu$. (Obviously in any case $\varphi \ll \nu)$.

Proof. Let $\mathscr{P}=\left\{E_{1}, \cdots, E_{n}\right\}$ be a (measurable) partition of $E \in \mathscr{A}$ and let $|\mathscr{P}|$ denote the number $\sum_{i=1}^{n}\left\|\varphi\left(E_{i}\right)\right\|$. Temporarily we will say that $E$ is unbounded if for each $K>0$ there is a partition $\mathscr{P}$ of $E$ with $|\mathscr{P}|>K$. Assume that $\varphi$ is $\sigma$-bounded on $E$, but 
that $E$ is unbounded. We claim that $E$ contains disjoint measurable subsets $E_{0}, E_{1}, \cdots, E_{n}, n \geqq 1$ with $E_{0}$ unbounded and $\sum_{i=1}^{n}\left\|\varphi\left(E_{i}\right)\right\|>1$. Otherwise each partition of $E$ contains precisely one unbounded set and for positive integer $n$ there is a partition $\mathscr{P}_{n}$ with $|\mathscr{P}| \geqq n+1$, containing the unbounded set $F_{n}$ for which we must have $\left\|\varphi\left(F_{n}\right)\right\| \geqq n$. If necessary, by refining these partitions we may obtain that $F_{n+1} \supseteqq F_{n}$ for each $n$. Since $F_{n}=F \cup \bigcup_{k=1}^{\infty}\left(F_{k} \backslash F_{k+1}\right)$, where $F=\bigcap_{k=1}^{\infty} F_{k}$, and $\varphi$ is $\sigma$-additive in norm, we have

$$
n \leqq\left\|\varphi\left(F_{n}\right)\right\| \leqq\|\varphi(F)\|+\sum_{k=n}^{\infty}\left\|\varphi\left(F_{k} \backslash F_{k+1}\right)\right\|
$$

which is impossible since $\sum_{k=1}^{\infty}\left\|\varphi\left(F_{k} \backslash F_{k+1}\right)\right\|$ is convergent, $E$ being $\sigma$-bounded. Having proved our claim, we arrive at a new contradiction, since then we may construct a disjoint sequence $\left\{E_{n}\right\}_{n=1}^{\infty}$ measurable of subsets of $E$ with $\sum_{n=1}^{\infty}\left\|\varphi\left(E_{n}\right)\right\|=\infty$. Thus a $\sigma$-bounded set $E$ is not unbounded, i.e., there is a constant $K_{E}>0$ such that $\sum_{n=1}^{\infty}\left\|\varphi\left(E_{n}\right)\right\|<K_{E}$ for each disjoint sequence $\left\{E_{n}\right\}_{n=1}$ of measurable subsets of $E$.

Now we define $\nu$ on $\mathscr{A}$ by $\nu(E)=\sup \left\{\sum_{n=1}^{\infty}\left\|\varphi\left(E_{n}\right)\right\|:\left\{E_{n}\right\}_{n=1}^{\infty} \subset \mathscr{A}\right.$, disjoint and $\left.\bigcup_{n=1}^{\infty} E_{n}=E\right\}$. Obviously $\|\varphi(E)\| \leqq \nu(E), \nu(E)<\infty$ if and only if $\varphi$ is $\sigma$-bounded on $E$, and $\varphi \ll \mu$ implies $\nu \ll \mu$. We only need to prove that $\nu$ is $\sigma$-additive. Suppose that $E=\bigcup_{n=1}^{\infty} E_{n}$ where the $E_{n}$ are disjoint and measurable. For any $\varepsilon>0$ there is a disjoint sequence $\left(G_{m}\right)_{m=1}^{\infty}$ of measurable subsets of $E$ such that $E=\bigcup_{m=1}^{\infty} G_{m}$ and $\nu(E) \leqq \sum_{m=1}^{\infty}\left\|\varphi\left(G_{m}\right)\right\|+\varepsilon$ (if $\nu(E)=\infty, E$ is not $\sigma$-bounded and the $G_{m}$ may taken such that $\left.\sum_{m=1}^{\infty}\left\|\varphi\left(G_{m}\right)\right\|=\infty\right)$. Since

$$
\varphi\left(G_{m}\right)=\sum_{n=1}^{\infty} \varphi\left(G_{m} \cap E_{n}\right),
$$

we have $\left\|\varphi\left(G_{m}\right)\right\| \leqq \sum_{n=1}^{\infty}\left\|\varphi\left(G_{m} \cap E_{n}\right)\right\|$ and therefore

$$
\nu(E) \leqq \sum_{m, n}\left\|\varphi\left(G_{m} \cap E_{n}\right)\right\|+\varepsilon \leqq \sum_{n=1}^{\infty} \nu\left(E_{n}\right)+\varepsilon .
$$

On the other hand, for each positive $n$ there is a disjoint sequence $\left\{G_{n m}\right\}_{m=1}^{\infty}$ of measurable sets such that $\bigcup_{m=1}^{\infty} G_{n m}=F_{n}$ and

$$
\mathcal{\nu}\left(E_{n}\right) \leqq \sum_{m=1}^{\infty}\left\|\varphi\left(G_{n m}\right)\right\|+2^{-n} \varepsilon .
$$

Then $\sum_{n=1}^{\infty} \nu\left(E_{n}\right) \leqq \sum_{n, m}\left\|\varphi\left(G_{n m}\right)\right\|+\varepsilon \leqq \nu(E)+\varepsilon$. Since $\varepsilon$ was arbitrary, we obtain $\nu(E)=\sum_{n=1}^{\infty} \nu\left(E_{n}\right)$.

2.3. Lemma. Let $f(\omega)$ and $r(\omega)$ be integrable functions, the first complex and the second nonnegative, such that for each $E \in \mathscr{A}$, $\left|\int_{E} f(\omega) d \mu(\omega)\right| \leqq \int_{E} r(\omega) d \mu(\omega)$. Then $|f(\omega)| \leqq r(\omega)$ almost everywhere. 
Proof. If the lemma is false, there is a positive integer $n$ such that $\mu(\{\omega \in \Omega:|f(\omega)|>r(\omega)+1 / n\})>0$ since then $\{\omega \in \Omega:|f(\omega)|>r(\omega)\}$ has positive measure. Also, for some open circle $S$ of radius $1 / 2 n$ on the complex plane we must have $0<\mu(F)<\infty$, where $F$ denotes a subset of $\{\omega:|f(\omega)|>r(\omega)+1 / n\} \cap\{\omega: f(\omega) \in S\}$. Let $z_{0}$ be center of $S$. Then for each $\omega \in F,\left|f(\omega)-z_{0}\right|<1 / 2 n$ and $|f(\omega)|>r(\omega)+1 / n$. Integrating the identity $f(\omega)=z_{0}-\left(z_{0}-f(\omega)\right)$ over $F$ and taking absolute values we obtain

$$
\begin{aligned}
\left|\int_{F} f(\omega) d \mu(\omega)\right| & \geqq\left|\int_{F} z_{0} d \mu(\omega)\right|-\left|\int_{F}\left(z_{0}-f(\omega)\right) d \mu(\omega)\right| \\
& \geqq\left|z_{0}\right| \mu(F)-1 / 2 n \mu(F)>r(\omega) \mu(F)
\end{aligned}
$$

for all $\omega \in F$, since $r(\omega)<|f(\omega)|-1 / n<1 / 2 n \div\left|z_{0}\right|-1 / n$. Integrating again over $F$ and dividing by $\mu(F)$ we obtain

$$
\left|\int_{F} f(\omega) d \mu(\omega)\right|>\int_{F} r(\omega) d \mu(\omega)
$$

which contradicts our hypothesis.

2.4. TheOREM. Let $\phi$ be a measure defined on $\mathscr{A}$ and taking values in $H$ or $B(H)$. If $\phi$ is $\sigma$-additive in norm, $\sigma$-bounded and absolutely continuous with respect to $\mu$ then $\phi$ is the indefinite integral with respect to $\mu$ of an integrable vector function or operator function which is unique almost everywhere.

Proof. We consider first the case in which $\varphi$ takes values in $H$. Since for each $z \in H,(x, \varphi(E))$ is a complex, finite measure, absolutely continuous with respect to $\mu$, the Radon-Nikodým theorem says that there is a complex integrable function $f_{\omega}(x)$ (with respect to $\omega$ ) such that

$$
(x, \varphi(E))=\int_{E} f_{\omega}(x) d \mu(\omega)
$$

and the function $f_{\omega}(x)$ differs from another with the same properties at most in a $\mu$-null set. If $\alpha, \beta$ are complex and $x, y \in H$, it is clear that $f_{\omega}(\alpha x+\beta y)=\alpha f_{\omega}(x)+\beta f_{\omega}(y)$ except in a $\mu$-null set. Also

$$
\left|\int_{E} f_{\omega}(x) d \mu(\omega)\right|=|(x, \varphi(E))| \leqq\|\varphi(E)\| \cdot\|x\| \leqq \nu(E)\|x\|,
$$

where $\nu$ is the measure defined in Lemma 2.2. Since $\nu \ll \mu$ and $\nu$ is finite, there is a nonnegative, finite and integrable function $r_{\omega}$ such that $\nu(E)=\int_{E} r_{\omega} d \mu(\omega)$. From the inequality

$$
\left|\int_{E} f_{\omega}(x) d \mu(\omega)\right| \leqq \int_{E} r_{\omega}\|x\| d \mu(\omega)
$$


for each $E \in \mathscr{A}$, by Lemma 2.3. we conclude that $\left|f_{\omega}(x)\right| \leqq r_{\omega}\|x\|$ for almost all $\omega$.

The next steps of the proof lead to the construction for each $x \in H$ of a particular function $f_{\omega}(x)$, which for each $\omega$ will be a continuous linear functional in $x$. Let $\left\{e_{1}, e_{2}, \cdots\right\}$ be an orthonormal base for $H$ and let $H_{0}$ be the set of linear combinations with rational complex coefficients of the base vectors.

Step 1. We choose finite functions $\widetilde{f}_{\omega}\left(e_{k}\right)$ such that $\left(e_{k}, \varphi(E)=\right.$ $\int_{E} \tilde{f}_{\omega}\left(e_{k}\right) d \mu(\omega)$ for each $E \in \mathscr{A}$.

Step 2. We define $\widetilde{f}_{\omega}$ on $H_{0}$ by linearity.

Step 3. We choose a nonnegative, finite function $r_{\omega}$ such that $\nu(E)=\int_{E} r_{\omega} d \mu(\omega)$ for each $E \in \mathscr{A}$.

Step 4 . Since $H_{0}$ is countable and for each $x \in H_{0},\left|\tilde{f}_{\omega}(x)\right| \leqq r_{\omega}\|x\|$ for almost all $\omega$, we choose a $\mu$-null set $N$ such that $\left|\widetilde{f}_{\omega}(x)\right| \leqq r_{\omega}\|x\|$ for all $x \in H_{0}$ and $\omega \in \Omega \backslash N$.

Step 5. We define $f_{\omega}(x)$ for $\omega \in \Omega$ and $x \in H_{0}$ by $f_{\omega}(x)=\tilde{f}_{\omega}(x)$ if $\omega \in \Omega \backslash N$ and $f_{\omega}(x)=0$ if $\omega \in N$. The functions we have defined have the following properties:

(a) $(x, \varphi(E))=\int_{E} f_{\omega}(x) d \mu(\omega)$, for each ${ }^{-} x \in H_{0}$ ane $E \in \mathscr{A}$.

(b) $\left|f_{\omega}(x)\right| \leqq r_{\omega}\|x\|$ for each $x \in H_{0}$ and $\omega \in \Omega$,

(c) if $\alpha, \beta$ are rational complex numbers and $x, y \in H_{0}$, then $f_{\omega}(\alpha x+\beta y)=\alpha f_{\omega}(x)+\beta f_{\omega}(y)$, for all $\omega \in \Omega$.

Step 6. Let $x \in H$ and $\left\{x_{n}\right\}_{n=1}^{\infty}$ be a sequence in $H_{0}$ converging to $x$. For each $\omega \in \Omega,\left|f_{\omega}\left(x_{n}\right)-f_{\omega}\left(x_{m}\right)\right|=\left|f_{\omega}\left(x_{n}-x_{m}\right)\right| \leqq r_{\omega}|| x_{n}-x_{m} \|$. Therefore $\lim _{n \rightarrow \infty} f_{\omega}\left(x_{n}\right)$ exists and obviously it is independent of the particular sequence $\left\{x_{n}\right\}_{n=1}^{\infty}$. We define $f_{\omega}(x)=\lim _{n \rightarrow \infty} f_{\omega}\left(x_{n}\right)$. From the continuity of the norm we obtain $\left|f_{\omega}(x)\right| \leqq r_{\omega}\|x\|$. Also $(x, \varphi(E))=$ $\lim _{n \rightarrow \infty}\left(x_{n}, \varphi(E)\right)=\lim _{n \rightarrow \infty} \int_{E} f_{\omega}\left(x_{n}\right) d \mu(\omega)=\int_{E} f_{\omega}(x) d \mu(\omega)$, the last equality being valid by the dominated convergence theorem. Finally, if $\alpha, \beta$ are arbitrary complex numbers and $x, y$ are any two vectors in $H$, there are sequences $\left\{\alpha_{n}\right\}_{n=1}^{\infty},\left\{\beta_{n}\right\}_{n=1}^{\infty}$ of rational complex numbers and sequences $\left\{x_{n}\right\}_{n=1}^{\infty},\left\{y_{n}\right\}_{n=1}^{\infty}$ of vectors in $H_{0}$ such that $\lim _{n \rightarrow \infty} \alpha_{n}=\alpha$, $\lim _{n \rightarrow \infty} \beta_{n}=\beta, \lim _{n \rightarrow \infty} x_{n}=x, \lim _{n \rightarrow \infty} y_{n}=y$. Then $f_{\omega}(\alpha x+\beta y)=\lim _{n \rightarrow \infty}$ $f_{\omega}\left(\alpha_{n} x_{n}+\beta_{n} y_{n}\right)=\lim _{n \rightarrow \infty}\left(\alpha_{n} f_{\omega}\left(x_{n}\right)+\beta_{n} f_{\omega}\left(y_{n}\right)\right)=\alpha f_{\omega}(x)+\beta f_{\omega}(y)$.

Thus for each $\omega, f_{\omega}(x)$ is a continuous linear functional and by the Riesz theorem there is a unique vector $x(\omega)$ such that $f_{w}(x)=$ $(x, x(\omega))$ for each $x \in H$. Since $f_{\omega}(x)$ is measurable, $x(\omega)$ is measurable and since $\|x(\omega)\|=\left\|f_{\omega}\right\| \leqq r_{\omega}, x(\omega)$ is also integrable. From the equation $(x, \varphi(E))=\int_{E}(x, x(\omega)) d \mu(\omega)=\left(x, \int_{E} x(\omega) d \mu(\omega)\right)$ we obtain $\varphi(E)=$ $\int_{E} x(\omega) d \mu(\omega)$. The uniqueness almost everywhere of the vector function $x(\omega)$ is trivial. 
The proof for the case when $\varphi$ takes values in $B(H)$ follows along the same lines. Now we obtain $(\varphi(E) x, y)=\int_{E} f_{\omega}(x, y) d \mu(\omega)$, where $f_{\omega}(x, y)$ is for all $x, y \in H$ an integrable function and for each $\omega \in \Omega$ is a bilinear functional in $x, y$, bounded by some Radon-Nikodým derivative $r_{\omega}$ of the measure $\nu$. By a corollary of the Riesz theorem, $f_{\omega}(x, y)=(A(\omega) x, y)$ for some linear operator $A(\omega)$, with $\|A(\omega)\|=$ $\left\|f_{\omega}\right\| \leqq r_{\omega}$ and as before we obtain $\varphi(E)=\int_{E} A(\omega) d \mu(\omega)$ for each $E \in \mathscr{A}$. The uniqueness a.e. of $A(\omega)$ is again trivial.

2.5. Remark. From the proof of Theorem 2.4., we have that $\|x(\omega)\| \leqq r_{\omega}$ (a.e.), where $r_{\omega}=d \nu / d \mu$ (a.e.). It is easy to see that $\|x(\omega)\|$ is actually equal to $r_{\omega}$ (a.e.). In fact, from $\|\varphi(E)\| \leqq \nu(E)$ and the definition of $\nu(E)$, we obtain $\int_{E}\|x(\omega)\| d \mu \geqq \nu(E)$ since

$$
\sum_{n=1}^{\infty}\|\varphi(E)\| \leqq \sum_{n=1}^{\infty} \int_{E}\|x(\omega)\| d \mu=\int_{E_{n}}\|x(\omega)\| d \mu
$$

for each countable partition of $E$. Also $\int_{E}\|x(\omega)\| d \mu \leqq \int_{E} r_{\omega} d \mu=\nu(E)$ and therefore $\|x(\omega)\|=r_{\omega}$ (a.e.). If we write $x(\omega)=d \varphi / d \mu, r_{\omega}=$ $d \nu / d \mu$, we have $\|d \varphi / d \mu\|=d \nu / d \mu$. Of course, the same formula holds for operator valued measures.

2.6. If $x(\omega)$ is a measurable function which is not necessarily integrable, we may still integrate it on those sets in $\mathscr{A}$ where $\|x(\omega)\|$ is integrable. In fact, since $\|x(\omega)\|$ is everywhere finite and $\mu$ is $\sigma$ finite, there is a countable covering of $\Omega$ consisting of such sets. On each of these sets the indefinite integral is $\sigma$-bounded. Reciprocally, if there is a countable covering of $\Omega$ by measurable sets $\Omega_{n}$ and a vector (or operator) valued measure $\varphi$ defined on the measurable subsets of each $\Omega_{n}$, which is $\sigma$-additive and $\sigma$-bounded on each $\Omega_{n}$, then $\varphi$ is the indefinite integral of some unique (a.e.) $\mathscr{A}$-measurable vector (or operator) function, and this function will be integrable if and only if the (unique) extension of $\phi$ to all of $\mathscr{A}$, is $\sigma$-additive in norm and $\sigma$-bounded.

2.7. A CountereXAMPLe. We may exhibit a vector (or operator) measure $\varphi$ which is $\sigma$-additive on $\mathscr{A}$, absolutely continuous with respect to some non-negative measure $\mu$, but $\sigma$-bounded only on sets of $\mu$-measure zero. In fact there is a vector measure $\gamma$ defined on the Borel subsets of $[0,1]$, such that for each Borel set $E,\|\gamma(E)\|=\sqrt{\lambda(E)}$, where $\lambda$ is the Lebesgue measure of $E$ (so that $\gamma \ll \lambda$ ), and furthermore, if $E_{1} \cap E_{2}=\varnothing$ then $\left(\gamma\left(E_{1}\right), \gamma\left(E_{2}\right)\right)=0$, i.e., $\gamma\left(E_{1}\right)$ and $\gamma\left(E_{2}\right)$ are 
orthogonal. It is easy to see that such a measure is $\sigma$-additive in norm, absolutely continuous with respect to $\lambda$, and if $\gamma(E) \neq 0$ (or equivalently, $\lambda(E) \neq 0$ ), then $\gamma$ is not $\sigma$-bounded on $E$.

In fact, let $\mathscr{S}$ denote the Borel sets on $[0,1]$ and let $\left\{E_{k}\right\}_{k=1}^{\infty}$ be a disjoint sequence in $\mathscr{B}, \bigcup_{k=1}^{\infty} E_{k}=E$. Then $\left\|\gamma(E)=\sum_{k=1}^{n} \gamma\left(E_{k}\right)\right\|=$ $\left\|\gamma(E)-\gamma\left(\bigcup_{k=1}^{n} E_{k}\right)\right\|=\left\|\gamma\left(\bigcup_{k=n+1}^{\infty} E_{k}\right)\right\|=\sqrt{\lambda\left(\bigcup_{k=n+1}^{\infty} E_{k}^{\prime}\right)} \rightarrow 0$ as $n \rightarrow \infty$ and therefore $\gamma(E)=\sum_{k=1}^{\infty} \gamma\left(E_{k}\right)$.

Now let $\gamma(E) \neq 0$. Consider the sequence $\left\{t_{n}\right\}_{n=1}^{\infty}$ in $[0,1]$ defined by $t_{n}=\inf \left\{t: \lambda(E \cap[0, t])>6 \lambda(E) / \pi^{2} \sum_{k=1}^{n} 1 / k^{2}\right\}$ for $n \geqq 1$ and $t_{0}=0$. We define $E_{n}=E \cap\left[t_{n-1}, t_{n}\right]$ so that $\left\{E_{n}\right\}_{n=1}^{\infty}$ is a disjoint sequence in and $\bigcup_{n=1}^{\infty} E_{n} \subseteq E$. Also $\lambda\left(E_{n}\right)=6 \lambda(E) / \pi^{2} n^{2}$ and therefore

$$
\left\|\gamma\left(E_{n}\right)\right\|=\frac{\sqrt{6 \lambda(E)}}{\pi} \cdot \frac{1}{\pi}
$$

so that $\sum_{n=1}^{\infty}\left\|\gamma\left(E_{n}\right)\right\|$ diverges, although $\sum_{n=1}^{\infty} \gamma\left(E_{n}\right)$ is obviously convergent and equal to $\gamma(E)$. (Let $E_{0}=E \backslash \bigcup_{n=1}^{\infty} E_{n}$, then $\lambda\left(E_{0}\right)=0$ and therefore $\gamma(E)=0)$.

2.8. Construction of $\gamma$. We construct first inductively a sequence of sets $\left\{A_{n}\right\}_{n=1}^{\infty}$ in $H$ having the following properties:

(i) $A_{n}$ consists of $2^{n}$ mutually orthogonal vectors $a_{n}^{1}, a_{n}^{2}, \cdots, a_{n}^{2 n}$ each of length $2^{-n / 2}$.

(ii) For each $n \geqq 0$ and $1 \leqq p \leqq 2^{n}, a_{n}^{p}=a_{n+1}^{2 p-1}+a_{n+1}^{2 p}$.

We start choosing a unit vector which we denote by $a_{0}^{1}$ and call $A_{0}=\alpha_{0}^{1}$. Having constructed $A_{0}, A_{1}, \cdots, A_{n}$, we construct $A_{n+1}$ in the following way. Choose $2^{n}$ vectors $b_{1}, b_{2}, \cdots, b_{2^{n}}$, each of length $2^{-n / 2}$, orthogonal with respect to each other and to $a_{n}^{1}, a_{n}^{2}, \cdots, a_{n}^{2 n}$. Now define $a_{n+1}^{2 p-1}=1 / 2\left(a_{n}^{p}+b_{p}\right), a_{n+1}^{2 p}=1 / 2\left(a_{n}^{p}-b_{p}\right), p=1,2, \cdots, 2^{n}$ and then $A_{n+1}=\left\{a_{n+1}^{1}, a_{n+1}^{2}, \cdots, a_{n+1}^{2 n+1}\right\}$. Obviously a sequence $\left\{A_{n}\right\}_{n=1}^{\infty}$ constructed in this way satisfies (i-ii).

Now we begin the construction of our measure. A basic interval of order $n$ will be an interval of the form $\left[p-1 / 2^{n}, p / 2^{n}\right]$ where $n$ and $p$ are integers and $n \geqq 0,1 \leqq p \leqq 2^{n}$. $\mathscr{F}$ and $\mathscr{G}$ will denote respectively the class of all finite unions and the class of all countable unions of basic intervals and $\mathscr{S}$ will denote the Borel sets of $[0,1)$. A set in $\mathscr{F}$ (or in $\mathscr{C}$ ) can always be expressed as a finite (or countable) union of disjoint basic intervals. For a set in $\mathscr{F}$ this is obvious and for a set in $\mathscr{G}$ a simple inductive process will give us the required decomposition. It is clear that $\mathscr{F}$ is an algebra, that is, it is closed with respect to finite unions and complementation. $\mathscr{G}$ is closed with respect to countable unions and finite intersections. The latter follows from the identity $\left(\bigcup_{j=1}^{\infty} F_{i}\right) \cap\left(\bigcup_{j=1}^{\infty} H_{j}\right)=\bigcup_{i=1}^{\infty}\left(F_{i} \cap H_{i}\right)$, where $\left\{F_{i}\right\}_{i=1}^{\infty}$ and $\left\{H_{i}\right\}_{i=1}^{\infty}$ are nondecreasing sequences of sets in $\mathscr{F}$. 
If $V$ is the basic interval [ $p-1 / 2^{n}, p / 2^{n}$ ), we define $\gamma(V)=a_{n}^{p}$. If $V_{1}=\left[2 p-2 / 2^{n+1}, 2 p-1 / 2^{n+1}\right)$ and $V_{2}=\left[2 p-1 / 2^{n+1}, 2 p / 2^{n+1}\right)$, so that $V=V_{1} \cup V_{2}$, by (ii) we have that $\gamma(V)=\gamma\left(V_{1}\right)+\gamma\left(V_{2}\right)$. By induction we obtain that if $V_{1}, V_{2}, \cdots, V_{2^{m}}$ denote the $2^{m}$ basic subintervals of $V$ of order $n+m$, then $\gamma(V)=\sum_{n=1}^{\infty} \gamma\left(V_{i}\right)$. Finally if $V_{1}, V_{2}, \cdots, V_{n}$ are disjoint basic intervals, not necessarily of the same order, such that $V=\bigcup_{i=1}^{n} V_{i}$ and $n+m$ is the highest order among the $V_{i}$, we decompose each $V_{i}$ in basic subintervals of order $n+m$, say $V_{i}=$ $\bigcup_{i} W_{j}^{(i)}$, so that $\gamma\left(V_{i}\right)=\sum_{i} \gamma\left(W_{j}^{(i)}\right)$ and we obtain

$$
\sum_{i=1}^{n} \gamma\left(V_{i}\right)=\sum_{i} \sum_{j} \gamma\left(W_{j}^{(i)}\right)=\gamma(V) .
$$

Thus $\gamma$ is additive on the basic intervals.

If $F \in \mathscr{F}$ and $F=\bigcup_{i=1}^{n} V_{i}$, where the $V_{i}$ are disjoint basic intervals, we define $\gamma(F)=\sum_{i=1}^{n} \gamma\left(V_{i}\right)$. From the additivity of $\gamma$ on the basic intervals it follows immediately that $\gamma(F)$ is well defined, i.e., it doesn't depend upon the particular decomposition of $F$ and that $\gamma$ is additive on $\mathscr{F}$.

If $V=\left[p-1 / 2^{n}, p / 2^{n}\right),\|\gamma(V)\|^{2}=\left\|a_{n}^{p}\right\|^{2}=\| 2^{-n}=\lambda(V)$, where $\lambda$ denotes Lebesgue measure. If $V_{1}$ and $V_{2}$ are disjoint basic intervals, $\gamma_{2}\left(V_{1}\right)$ and $\gamma\left(V_{2}\right)$ are mutually orthogonal, which implies that $\|\gamma(F)\|^{2}=$ $\left\|\sum_{i=1}^{n} \gamma\left(V_{i}\right)\right\|^{2}=\sum_{i=1}^{n}\left\|\gamma\left(V_{i}\right)\right\|^{2}=\sum_{i=1}^{n} \lambda\left(V_{i}\right)=\lambda(F)$, where $F \in \mathscr{F}, F=$ $\bigcup_{i=1}^{n} V_{i}$ and $V_{i}$ are disjoint basic intervals.

Suppose now that $V=\bigcup_{i=1}^{\infty} V_{i}$, where the $V_{i}$ are disjoint basic intervals and $V$ is also a basic interval. Then $V \backslash \bigcup_{i=1}^{n} V_{i} \in \mathscr{F}$ for each $n \geqq 1$ and therefore $\left\|\gamma(V)-\sum_{i=1}^{n} \lambda\left(V_{i}\right)\right\|=\left\|\gamma\left(V \backslash \bigcup_{i=1}^{n} V_{i}\right)\right\|=$

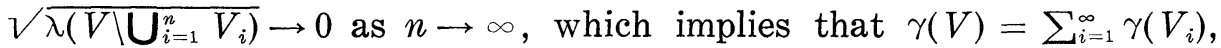
i.e., $\gamma$ is $\sigma$-additive on the basic intervals.

Now we define $\gamma$ on $\mathscr{C}$ by $\gamma(G)=\sum_{i=1}^{\infty} \gamma\left(V_{i}\right)$, where $G=\bigcup_{i=1}^{\infty} V_{i}$ and the $V_{i}$ are disjoint basic intervals. First we observe that since the vector $\gamma\left(V_{i}\right)$ are pairwise orthogonal and $\sum_{i=1}^{\infty}\left\|\gamma\left(V_{i}\right)\right\|^{2}=\sum_{i=1}^{\infty} \gamma\left(V_{i}\right)=$ $\lambda(G) \leqq 1$, the series $\sum_{i=1}^{\infty} \gamma\left(V_{i}\right)$ converges and $\|\gamma(G)\|^{2}=\lambda(G)$. If $G=$ $\bigcup_{i=1}^{\infty} V_{i}=\bigcup_{j=1}^{\infty} W_{j}$ are two decompositions of $G$ into disjoint basic subintervals, $\sum_{i=1}^{\infty} \gamma\left(V_{i}\right)=\sum_{i=1}^{\infty} \sum_{j=1}^{\infty} \gamma\left(V_{i} \cap W_{j}\right)=\sum_{j=1}^{\infty} \sum_{i=1}^{\infty} \gamma\left(V_{i} \cap W_{j}\right)=$ $\sum_{j=1}^{\infty} \gamma\left(W_{j}\right)$ (the sums commute because the vectors are orthogonal) so that $\gamma(G)$ is well defined. If $\left\{F_{i}\right\}_{i=1}^{\infty}$ is a nondecreasing sequence in $\mathscr{F}$ with $G=\bigcup_{n=1}^{\infty} F_{n}$, then $\gamma(G) \lim _{n \rightarrow \infty} \gamma\left(F_{n}\right)$. In fact there is a sequence $\left\{V_{i}\right\}_{i=1}^{\infty}$ of disjoint basic intervals such that $F_{n}=\bigcup_{i=1}^{r_{n}} V_{i}$, where $r_{1} \leqq r_{2} \leqq \cdots$ are integers with $\lim _{n \rightarrow \infty} r_{n}=\infty$, so that $\gamma(G)=$ $\lim _{n \rightarrow \infty} \sum_{i=1}^{n} \gamma\left(V_{i}\right)=\lim _{n \rightarrow \infty} \sum_{i=1}^{r_{n}} \gamma\left(V_{i}\right)=\lim _{r \rightarrow \infty} \gamma\left(F_{n}\right)$. Suppose now that $G_{1}$ and $G_{2}$ are in $\mathscr{C}$ and that $\left\{F_{n}\right\}_{n=1}^{\infty},\left\{H_{n}\right\}_{n=1}^{\infty}$ are nondecreasing sequences in $\mathscr{F}$ with $G_{1}=\bigcup_{n=1}^{\infty} F_{n}, G_{2}=\bigcup_{n=1}^{\infty} H_{n}$. Then we have that $G_{1} \cup G_{2}=\bigcup_{n=1}^{\infty}\left(F_{n} \cup H_{n}\right), G_{1} \cap G_{2}=\bigcup_{n=1}^{\infty}\left(F_{n} \cap H_{n}\right)$, and taking limits, from the relation $\gamma\left(F_{n} \cup H_{n}\right)+\gamma\left(F_{n} \cap H_{n}\right)=\gamma\left(F_{n}\right)+\gamma\left(H_{n}\right)$ we obtain 
$\gamma\left(G_{1} \cup G_{2}\right)+\gamma\left(G_{1} \cap G_{2}\right)=\gamma\left(G_{1}\right)+\gamma\left(G_{2}\right)$, i.e., $\gamma$ is modular in $\mathscr{G}$.

It is clear that $\mathscr{G}$ contains all open sets in $[0,1)$. Therefore, if $E \in \mathscr{B}$, for each $\varepsilon>0$, there is some $G \in \mathscr{G}$ such that $G \supseteqq E$ and $\lambda(G \backslash E)<\varepsilon$. Let $G_{1}, G_{2} \in \mathscr{C}, G_{1} \subseteq G_{2}, G_{1}=\bigcup_{i=1}^{\infty} V_{i}$, the $V_{i}$ disjoint basic intervals. Then for each $n, G_{2} \mid \bigcup_{i=1}^{n} V_{i} \in \mathscr{C}$ and expressing $G_{2} \mid \bigcup_{i=1}^{n} V_{i}$ as a union of disjoint basic intervals we see that $\gamma\left(G_{2} \mid \bigcup_{i=1}^{n} V_{i}\right)$ $\gamma\left(G_{2}\right)-\sum_{i=1}^{n} \gamma\left(V_{i}\right)$. Therefore

$$
\begin{aligned}
& \left\|\gamma\left(G_{2}\right)-\gamma\left(G_{1}\right)\right\|^{2}=\lim _{n \rightarrow \infty}\left\|\gamma\left(G_{2}\right)-\sum_{i=1}^{n} \gamma\left(V_{i}\right)\right\|^{2} \\
& \left.\quad=\lim _{n \rightarrow \infty} \| \gamma\left(G_{2}\right) \bigcup_{i=1}^{n} V_{i}\right) \|^{2}=\lim _{n \rightarrow \infty} \gamma\left(G_{2} \mid \bigcup_{i=1}^{n} V_{i}\right)=\lambda\left(G_{2}\right)-\lambda\left(G_{1}\right) .
\end{aligned}
$$

This implies that if the sequence $\left\{G_{n}\right\}_{n=1}^{\infty}$ of sets in $\mathscr{C}$ is nonincreasing, each $G_{n}$ contains $G \in \mathscr{B}$ and $\lim _{n \rightarrow \infty} \lambda\left(G_{n}\right)=\lambda(E)$, then $\left\{\gamma\left(G_{n}\right)\right\}_{n=1}^{\infty}$ is a Cauchy sequence in $H$. We define $\gamma(E)$ as the limit of this sequence and obviously $\|\gamma(E)\|^{2}=\gamma(E)$. In order to prove that $\gamma(E)$ does not depend upon the particular sequence $\left\{G_{n}\right\}_{n=1}^{\infty}$, we take another such sequence, say $\left\{\widetilde{G}_{n}\right\}_{n=1}^{\infty}$. Evidently $\lim _{n \rightarrow \infty} \lambda\left(G_{m} \mid \widetilde{G}_{n}\right)=\lim _{n \rightarrow \infty} \lambda\left(\widetilde{G}_{n} \mid G_{n}\right)=0$ and since

$$
\begin{aligned}
& \left\|\gamma\left(G_{n}\right)-\gamma\left(\widetilde{G}_{n}\right)\right\| \leqq\left\|\gamma\left(G_{n}\right)-\gamma\left(G_{n} \cap \widetilde{G}_{n}\right)\right\| \\
& \quad+\left\|\gamma\left(\widetilde{G}_{n}\right)-\gamma\left(G_{n} \cap \widetilde{G}_{n}\right)\right\|=\sqrt{\lambda\left(G_{n} \backslash \widetilde{G}_{n}\right)}+\sqrt{\lambda\left(\widetilde{G}_{n} \backslash G_{n}\right)},
\end{aligned}
$$

we have $\lim _{n \rightarrow \infty}\left\|\gamma\left(G_{n}\right)-\gamma\left(\widetilde{G}_{n}\right)\right\|=0$ and therefore $\lim _{n \rightarrow \infty} \gamma\left(G_{n}\right)=\lim _{n \rightarrow \infty} \gamma\left(\widetilde{G}_{n}\right)$. If $G \in \mathscr{G}$ and $G \supseteqq E, E \in \mathscr{B}$, there is a nonincreasing sequence $\left\{G_{n}\right\}_{n=1}^{\infty}$ of sets in $\mathscr{C}, G \supseteqq G_{n}$ and such that $\gamma(E)=\lim _{n \rightarrow \infty} \gamma\left(G_{n}\right)$. Then $\|\gamma(G)-\gamma(E)\|^{2}=\lim _{n \rightarrow \infty}\left\|\gamma(G)-\gamma\left(G_{n}\right)\right\|^{2}=\lim _{n \rightarrow \infty} \lambda\left(G \backslash G_{n}\right)=\lambda(G \backslash E)$.

Our next step is to show that $\gamma$ is finitely additive in $\mathscr{B}$. Let $E_{1}$ and $E_{2}$ be disjoint sets in $\mathscr{B}$ and let $G_{1}$ and $G_{2}$ in $\mathscr{G}$ be such that $G_{1} \supseteqq E_{1}, G_{2} \supseteqq E_{2},\left\|\gamma\left(G_{1}\right)-\gamma\left(E_{1}\right)\right\|<\varepsilon$ and $\left\|\gamma\left(G_{2}\right)-\gamma\left(E_{2}\right)\right\|<\varepsilon$, where $\varepsilon>0$ is given. Then

$$
\begin{aligned}
& \left\|\gamma\left(G_{1} \cup G_{2}\right)-\gamma\left(E_{1} \cup E_{2}\right)\right\| \\
& \quad=\sqrt{\lambda\left(G_{1} \cup G_{2}\right)-\lambda\left(E_{1}^{\prime} \cup E_{2}\right)} \leqq \sqrt{\lambda\left(G_{1} \backslash E_{1}\right)+\lambda\left(G_{2} \backslash E_{2}\right)}<\sqrt{2 \varepsilon} .
\end{aligned}
$$

Also since $\gamma$ is modular in $\mathscr{C}$,

$$
\begin{aligned}
& \left\|\gamma\left(G_{1} \cup G_{2}\right)-\gamma\left(G_{1}\right)-\gamma\left(G_{2}\right)\right\|=\left\|\gamma\left(G_{1} \cup G_{2}\right)\right\| \\
& \quad=\sqrt{\left.\lambda G_{1} \cap G_{2}\right)} \leqq \sqrt{\lambda\left(G_{1} \backslash E_{1}\right)+\lambda\left(G_{2} \backslash E_{2}\right)}<\sqrt{2 \varepsilon} .
\end{aligned}
$$

Therefore

$$
\begin{aligned}
& \left\|\gamma\left(E_{1} \cup E_{2}\right)-\gamma\left(E_{1}\right)-\gamma\left(E_{2}\right)\right\| \leqq\left\|\gamma\left(E_{1} \cup E_{2}\right)-\gamma\left(G_{1} \cup G_{2}\right)\right\| \\
& \quad+\left\|\gamma\left(G_{1} \cup G_{2}\right)-\gamma\left(G_{1}\right)-\gamma\left(G_{2}\right)\right\|+\left\|\gamma\left(G_{1}\right)-\gamma\left(E_{1}\right)\right\| \\
& \quad+\gamma\left(G_{2}\right)-\gamma\left(E_{2}\right) \|<(2+2 \sqrt{2}) \varepsilon,
\end{aligned}
$$


which implies that $\gamma\left(E_{1} \cup E_{2}\right)=\gamma\left(E_{1}\right)+\gamma\left(E_{2}\right)$.

In 2.7. we proved that $\gamma$ is countable additive under the assumption that it is finitely additive and $\|\gamma(E)\|^{2}=\lambda(E)$ for $E \in \mathscr{B}$. Thus $\gamma$ is countably additive.

Next, in order to prove the orthogonality property, we observe that since disjoint basic intervals have orthogonal measures, if $G_{1}$ and $G_{2}$ are disjoint sets in $\mathscr{C}, \gamma\left(G_{1}\right)$ and $\gamma\left(G_{2}\right)$ must be orthogonal. If $K_{1}$ and $K_{2}$ are disjoint compact sets, there are nonincreasing sequences $\left\{G_{n}\right\}_{n=1}^{\infty}$ and $\left\{\widetilde{G}_{n}\right\}_{n=1}^{\infty}$ of sets in $\mathscr{G}$ such that $G_{n} \cap \widetilde{G}_{m}=\varnothing$ for all $n$ and $m$, and $\lim _{n \rightarrow \infty} \gamma\left(G_{n}\right)=\gamma\left(K_{1}\right), \lim _{n \rightarrow \infty} \gamma\left(\widetilde{G}_{n}\right)=\gamma\left(K_{2}\right)$, which implies that $\gamma\left(K_{1}\right)$ and $\gamma\left(K_{2}\right)$ are orthogonal. Finally if $E_{1}$ and $E_{2}$ are disjoint sets in $\mathscr{B}$, there are nondecreasing sequences $\left\{K_{n}\right\}_{n=1}^{\infty},\left\{K_{n}\right\}_{n=1}^{\infty}$ of compact subsets of $E_{1}$ and $E_{2}$ such that $\lambda\left(E_{1}\right)=\lim _{n \rightarrow \infty} \lambda\left(E_{n}\right), \lambda\left(E_{2}\right)=\lim _{n \rightarrow \infty} \lambda\left(K_{n}\right)$, so that $\gamma\left(E_{1}\right)=\lim _{n \rightarrow \infty} \gamma\left(K_{n}\right), \gamma\left(E_{2}\right)=\lim _{n \rightarrow \infty} \gamma\left(K_{n}\right)$, and this implies that $\gamma\left(E_{1}\right)$ and $\gamma\left(E_{2}\right)$ are orthogonal. We may extend $\gamma$ to the Borel subsets of $[0,1]$ defining $\gamma(\{1\})=0$, and even "complete" it, defining $\gamma(E)=0$ if $E$ is a subset of a Borel set of $\lambda$-measure zero.

\section{BIBLIOGRAPHY}

1. S. Bochner, Integration von Funktionen, deren Wert die Elemente eines Vektorraumes sind, Fund. Math. 20 (1933), 262-276.

2. B. J. Pettis, On integration in vector spaces, Trans. Amer. Math. Soc. 44 (1938), $277-304$.

3. K. Yosida, Functional analysis, Springer-Verlag, Berlin, 1965.

Received April 20, 1967.

Universided DE Chile 



\section{Pacific Journal of Mathematics \\ Vol. 29, No. 1 \\ May, 1969}

Jorge Alvarez de Araya, A Radon-Nikodým theorem for vector and operator

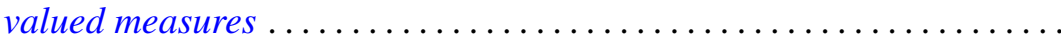

Deane Eugene Arganbright, The power-commutator structure of finite

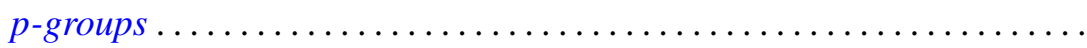

Richard Eugene Barlow, Albert W. Marshall and Frank Proschan, Some

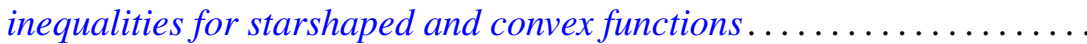

David Clarence Barnes, Some isoperimetric inequalities for the eigenvalues

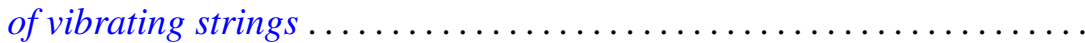

David Hilding Carlson, Critical points on rim-compact spaces ...........

Allan Matlock Weber Carstens, The lattice of pretopologies on an arbitrary

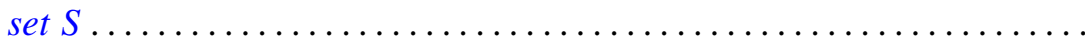

S. K. Chatterjea, A bilateral generating function for the ultraspherical

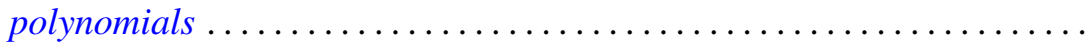

Ronald J. Ensey, Primary Abelian groups modulo finite groups ......... 77

Harley M. Flanders, Relations on minimal hypersurfaces ............ 83

Allen Roy Freedman, On asymptotic density in n-dimensions........... 95

Kent Ralph Fuller, On indecomposable injectives over artinian rings...... 115

George Isaac Glauberman, Normalizers of p-subgroups in finite groups . . . 137

William James Heinzer, On Krull overrings of an affine ring ........... 145

John McCormick Irwin and Takashi Ito, A quasi-decomposable abelian group without proper isomorphic quotient groups and proper isomorphic subgroups.

Allan Morton Krall, Boundary value problems with interior point boundary conditions

John S. Lowndes, Triple series equations involving Laguerre

polynomials

Philip Olin, Indefinability in the arithmetic isolic integers

Ki-Choul Oum, Bounds for the number of deficient values of entire functions whose zeros have angular densities..

R. D. Schafer, Standard algebras ....................

Wolfgang M. Schmidt, Irregularities of distribution. III.

Richard Alfred Tapia, An application of a Newton-like method to the Euler-Lagrange equation 